\title{
Chi-Square Mixture of Transformed/Inverse Transformed Gamma Family
}

\author{
Jayrold P. Arcede ${ }^{1}$, Miraluna L. Herrera ${ }^{1}$, Mark Jolet A. Cubelo ${ }^{1} \&$ Alfredo M. Griño ${ }^{2}$ \\ ${ }^{1}$ Mathematics Department, College of Arts and Sciences, Caraga State University, Butuan City, Philippines \\ ${ }^{2}$ UHLand Loan Corporation, Butuan City, Philippines \\ Correspondence: Jayrold P. Arcede, Mathematics Department, College of Arts and Sciences, Caraga State Univer- \\ sity, Butuan City 8600, Philippines. E-mail: jparcede@ carsu.edu.ph
}

Received: October 10, 2013 Accepted: October 27, 2013 Online Published: December 23, 2013

doi:10.5539/ijsp.v3n1p1 URL: http://dx.doi.org/10.5539/ijsp.v3n1p1

\begin{abstract}
In this study, we define the chi-square mixture of transformed gamma distribution which contained some special submodels namely, the chi-square mixture of gamma, Weibull, and exponential mixture distributions. Also, the chi-square mixture of inverse transformed gamma distribution is defined and a class of submodels are deduced, that is, the chi-square mixture of inverse gamma, inverse Weibull, and inverse exponential mixture distributions. For both classes, statistical properties are investigated, that is, mean, variance, skewness and kurtosis using $r$ th raw moment. The limiting behavior and special cases are also given to established relationships.
\end{abstract}

Keywords: mixture distribution, inverse/transformed gamma distribution, $r$ th raw moments, skewness, kurtosis

\section{Introduction}

Mixtures models have continued to receive increasing attention over the years from both practical and theoretical point of view. Fields in which mixture models have been successfully applied include, but not limited to, fisheries (Fleischman \& Burwen, 2003), economics (Alexander, 2004), medicine (Schlattmann, 2009), genetics (Schork et al., 1996), psychology (Ram \& Grimm, 2009), palaeontology (Hunt \& Chapman, 2001), archaeology (Dong, 1997), electrophoresis (Melnykov et al., 2011), sedimentology (Sylvester, 2007), geology (Coli et al., 2012), botany (Gutierrez et al., 1995), agriculture (Xu et al., 2010), zoology (Baral et al., 2013), communication theory (Yang \& Zwolinski, 2011) and engineering (Liu et al., 2008).

One of the oldest known applications of mixture model was that of Karl Pearson. Pearson (1894) successfully fitted a mixture of two univariate normal densities to the crab forehead breadth data provided by Weldon who speculated the presence of two new crab subspecies in the sample. Unfortunately, Pearson's method on moments suffered some computational difficulties where it involved solving of a ninth degree polynomial equation-an undaunted task at that time.

Mixture distribution occurs naturally when the population consists of several homogeneous subpopulations. In a finite mixture distribution, the density function is a convex combination of probability density functions (pdf) of the form $p_{i}(x \mid \theta)$ which can be represented as follows:

$$
m(x \mid \Theta)=\sum_{i=1}^{k} \pi_{i} p_{i}(x \mid \theta),
$$

where $0 \leq \pi_{i} \leq 1$ for $i=1,2, \ldots, k$ and $\sum_{i=1}^{k} \pi_{i}=1$. The pdf $p_{i}(x \mid \theta)$, called a component density, is the pdf of the $i$ th component for $i=1,2, \ldots, n$ associated with $\pi_{i}$ called a mixing weight. Moreover, $\Theta$ defines the set of parameter given as $\Theta=\left\{\pi_{1}, \pi_{2}, \ldots \pi_{n}, \theta_{1}, \theta_{2}, \ldots, \theta_{k}\right\}$.

Finite mixture distribution has been extensively studied (Böhning, 2007). An infinite analogue can also be formulated. If the parameter space $\Theta$ is absolutely continuous random variable having pdf $g(\theta)$, then we have a continuous mixture of densities $f(x \mid \theta)$ with weight function $g(\theta)$ of the following form:

$$
m(x \mid \Theta)=\int_{\Theta} f(x \mid \theta) g(\theta) d(\theta) .
$$


Recently, studies on infinite mixture distributions have surfaced (Adnan \& Kiser, 2012; Rezaul et al., 2011). In this article, we define the chi-square mixture distributions of transformed gamma and inverse transformed gamma distributions. This leads to a new family of chi-square mixture distribution that generalize the classical transformed/inverse transformed gamma family (Panjer, 2006). Furthermore, this study provides an analogue to similar studies done by Roy et al. (2005, 2006a, 2006b).

The rest of this article is organized as follows. Section 2 of the paper presents the mixtures of transformed Gamma family, mean, variance, skewness and kurtosis are obtained. Section 3 gives the mixtures of inverse transformed gamma family. Similary, mean, variance, skewness and kurtosis are obtained. The results and conclusions are given in Section 4.

\section{Mixture of Transformed Gamma Family}

This section presents the mixing of chi-square distribution with the transformed gamma family. Here, transformed gamma, Weibul, gamma and exponential distributions were considered as weight function. Relationships of the resulting mixture distributions are also shown. We have the following results.

Theorem 2.1 The chi-square mixture of exponential function given by

$$
f(x ; v, \theta)=\int_{0}^{\infty} \frac{e^{-\frac{\chi^{2}}{2}}\left(\chi^{2}\right)^{\frac{v}{2}-1}}{2^{\frac{v}{2}} \Gamma\left(\frac{v}{2}\right)} \frac{1}{\theta+\chi^{2}} e^{-\frac{x}{\theta+\chi^{2}}} d \chi^{2}, \quad 0<x<\infty
$$

where $\theta$ and $v$ are positive constants is a probability density function.

Proof. Function (3) is nonnegative, since the integrand is a product of two nonnegative functions, namely the chi-square and exponential distribution, and so integrating over $[0, \infty]$ becomes nonnegative.

It is sufficient to show that $\int_{-\infty}^{\infty} f(x ; v, \theta) d x=1$. Hence,

$$
\begin{aligned}
\int_{-\infty}^{\infty} f(x ; v, \theta) d x & =\int_{0}^{\infty} \int_{0}^{\infty} \frac{e^{-\frac{\chi^{2}}{2}}\left(\chi^{2}\right)^{\frac{v}{2}-1}}{2^{\frac{v}{2}} \Gamma\left(\frac{v}{2}\right)} \frac{1}{\theta+\chi^{2}} e^{-\frac{x}{\theta+\chi^{2}}} d \chi^{2} d x \\
& =\lim _{a \rightarrow \infty} \int_{0}^{a}\left[\lim _{b \rightarrow \infty} \int_{0}^{b} \frac{e^{-\frac{\chi^{2}}{2}}\left(\chi^{2}\right)^{\frac{v}{2}-1}}{2^{\frac{v}{2}} \Gamma\left(\frac{v}{2}\right)} \frac{1}{\theta+\chi^{2}} e^{-\frac{x}{\theta+\chi^{2}}} d x\right] d \chi^{2} \\
& =\lim _{a \rightarrow \infty} \int_{0}^{a} \frac{e^{-\frac{\chi^{2}}{2}}\left(\chi^{2}\right)^{\frac{v}{2}-1}}{2^{\frac{v}{2}} \Gamma\left(\frac{v}{2}\right)}\left[\lim _{b \rightarrow \infty} \int_{0}^{b} \frac{1}{\theta+\chi^{2}} e^{-\frac{x}{\theta+\chi^{2}}} d x\right] d \chi^{2} \\
& =1,
\end{aligned}
$$

where $\Gamma(\cdot)$ is the usual gamma function. The proof of Theorem 1 is now complete.

Definition 2.1 A random variable $X$ is said to have a chi-square mixture of exponential distribution with $v$ degrees of freedom and parameter $\theta$ if its density is given by function (3) above.

We now have the following theorem.

Theorem 2.2 Let $X$ be a random variable which follows a chi-square mixture of exponential distribution with $v$ degrees of freedom and parameter $\theta$. Then the rth raw moment about the origin is given by

$$
\mu_{r}^{\prime}=\Gamma(r+1) \int_{0}^{\infty} \frac{e^{-\frac{\chi^{2}}{2}}\left(\chi^{2}\right)^{\frac{v}{2}-1}}{2^{\frac{v}{2}} \Gamma\left(\frac{v}{2}\right)} \cdot\left(\theta+\chi^{2}\right)^{r} d \chi^{2}
$$

for $r>-1$. Furthermore, the mean is

$$
\theta+v
$$

and the variance is

$$
(\theta+v)^{2}+4 v
$$

The skewness, $\beta_{1}$, is

$$
\frac{\left[2 \theta^{3}+2 v^{3}+6 \theta v^{2}+24 v^{2}+6 \theta^{2} v+24 \theta v+48 v\right]^{2}}{\left[\theta^{2}+2 \theta v+v^{2}+4 v\right]^{3}}
$$


and the kurtosis, $\beta_{2}$, is

$$
\frac{9 \theta^{4}+9 v^{4}+36 \theta v^{3}+168 v^{3}+54 \theta^{2} v^{2}+336 \theta v^{2}+864 v^{2}+36 \theta^{3} v+168 \theta^{2} v+576 \theta v+1152 v}{\left[\theta^{2}+2 \theta v+v^{2}+4 v\right]^{2}} .
$$

Proof. Let $X$ be a random variable with density of chi-square mixture of exponential distribution. The $r$ th raw moment, $\mu_{r}^{\prime}$, is given by

$$
\begin{aligned}
\mu_{r}^{\prime} & =\int_{0}^{\infty} \int_{0}^{\infty} \frac{e^{-\frac{\chi^{2}}{2}} \chi^{2 \frac{v}{2}-1}}{2^{\frac{v}{2}} \Gamma\left(\frac{v}{2}\right)} \cdot\left(\theta+\chi^{2}\right)^{-1} e^{-\frac{x}{\left(\theta+\chi^{2}\right)}} x^{r} d x d \chi^{2} \\
& =\Gamma(r+1) \int_{0}^{\infty} \frac{e^{-\frac{\chi^{2}}{2}}\left(\chi^{2}\right)^{\frac{v}{2}-1}}{2^{\frac{v}{2}} \Gamma\left(\frac{v}{2}\right)} \cdot\left(\theta+\chi^{2}\right)^{r} d \chi^{2} .
\end{aligned}
$$

So, if $r=1$, the mean, $\mu_{1}^{\prime}$, is equal to

$$
\theta+v
$$

To solve the variance, let $r=2$ so that from Equation (4) we have

$$
\mu_{2}^{\prime}=2(\theta+v)^{2}+4 v
$$

Therefore the variance, $\mu_{2}$, is

$$
(\theta+v)^{2}+4 v
$$

In computing for skewness, $\beta_{1}$, given as:

$$
\frac{\mu_{3}^{2}}{\mu_{2}^{3}}
$$

we need to obtain the third central moment, $\mu_{3}$, as given by the formula:

$$
\mu_{3}^{\prime}-3 \mu_{2}^{\prime} \mu_{1}^{\prime}+2\left(\mu_{1}^{\prime}\right)^{3} .
$$

So for $r=3$, Equation (4) yields

$$
\begin{aligned}
\mu_{3}^{\prime} & =\Gamma(4) \int_{0}^{\infty} \frac{e^{-\frac{\chi^{2}}{2}}\left(\chi^{2}\right)^{\frac{v}{2}-1}}{2^{\frac{v}{2}} \Gamma\left(\frac{v}{2}\right)} \cdot\left(\theta+\chi^{2}\right)^{3} d \chi^{2} \\
& =3\left(\theta^{3}+6 \theta^{2}\left(\frac{v}{2}\right)+12 \theta\left(\frac{v}{2}\right)\left(\frac{v}{2}+1\right)+8\left(\frac{v}{2}+2\right)\left(\frac{v}{2}+1\right)\left(\frac{v}{2}\right)\right) \\
& =3 \theta^{3}+9 \theta^{2} v+9 \theta v^{2}+18 \theta v+3 v^{3}+18 v^{2}+24 v
\end{aligned}
$$

Hence,

$$
\mu_{3}^{\prime}=3 \theta^{3}+9 \theta^{2} v+9 \theta v^{2}+18 \theta v+3 v^{3}+18 v^{2}+24 v .
$$

By substituting Equation (5), (6) and (10) to Equation (9) we have

$$
\mu_{3}=6 v^{2}+24 v+6 \theta v-\left(\theta^{3}+3 \theta^{2} v+9 \theta v+7 v^{3}\right) .
$$

Therefore, by substituting Equations (7) and (11) to Equation (8) yields the following expression for skewness:

$$
\frac{\left[6 v^{2}+24 v+6 \theta v-\left(\theta^{3}+3 \theta^{2} v+9 \theta v+7 v^{3}\right)\right]^{2}}{\left(\theta^{2}+2 \theta v+3 v^{2}+4 v\right)^{3}} .
$$

Finally, we compute for kurtosis, $\beta_{2}$, as given by the formula:

$$
\frac{\mu_{4}}{\mu_{2}^{2}}
$$

First, we need the fourth central moment, $\mu_{4}$, given by

$$
\mu_{4}^{\prime}-4 \mu_{1}^{\prime} \mu_{3}^{\prime}+6 \mu_{2}^{\prime} \mu_{1}^{\prime 2}-3 \mu_{1}^{\prime 4} .
$$


We proceed by letting $r=4$, then Equation (4) becomes

$$
\mu_{4}^{\prime}=4 \theta^{4}+16 \theta^{3} v+24 \theta^{2} v^{2}+48 \theta^{2} v+16 \theta v^{3}+96 \theta v^{2}+128 \theta v+4 v^{4}+48 v^{3}+176 v^{2}+192 v .
$$

Consequently,

$$
\mu_{4}=\theta^{4}+4 \theta^{3} v+18 \theta^{2} v^{2}+28 \theta v^{3}-48 \theta v^{2}+32 \theta v+13 v^{4}+12 v^{2}+192 v .
$$

By substituting Equations (16) and (7) to Equation (13), we have the following expression for coefficient of kurtosis:

$$
\frac{\theta^{4}+4 \theta^{3} v+18 \theta^{2} v^{2}+28 \theta v^{3}-48 \theta v^{2}+32 \theta v+13 v^{4}+12 v^{2}+192 v}{\left(\theta^{2}+2 \theta v+3 v^{2}+4 v\right)^{2}}
$$

The proof is now complete.

As special case of this theorem: When $v \rightarrow 0$, the limiting distribution of chi-square mixture of exponential is the exponential distribution with parameter $\theta$.

In the succeding results, proofs are omitted as it follow similar idea in the preceding theorem.

Theorem 2.3 The chi-square mixture of Weibull function defined by

$$
f(x ; v, \theta, \tau)=\int_{0}^{\infty} \frac{e^{-\frac{\chi^{2}}{2}}\left(\chi^{2}\right)^{\frac{v}{2}-1}}{2^{\frac{v}{2}} \Gamma\left(\frac{v}{2}\right)} \frac{\tau\left(\frac{x}{\theta+\chi^{2}}\right)^{\tau} e^{-\left(\frac{x}{\theta+\chi^{2}}\right)^{\tau}}}{x} d \chi^{2}, \quad 0<x<\infty
$$

where parameters $\theta, \tau$ and $v$ are positive real numbers is a probability density function.

Definition 2.2 A random variable $X$ is said to have a chi-square mixture of Weibull distribution with parameters $\theta, \tau$ and $v$ if it has a density function given by (17) above.

We now have the following result.

Theorem 2.4 Let $X$ be a random variable which follows a chi-square mixture of Weibull distribution with parameters $v, \theta$ and $\tau$. Then the rth raw moment about the origin is given by,

$$
\mu_{r}^{\prime}=\mathbb{E}\left[X^{r}\right]=\Gamma\left(1+\frac{r}{\tau}\right) \int_{0}^{\infty} \frac{e^{-\frac{\chi^{2}}{2}}\left(\chi^{2}\right)^{\frac{v}{2}-1}}{2^{\frac{v}{2}} \Gamma\left(\frac{v}{2}\right)}\left(\theta+\chi^{2}\right)^{r} d \chi^{2},
$$

for $\tau>-r$. Furthermore the mean, $\mu_{1}^{\prime}$, is

$$
\Gamma\left(1+\frac{1}{\tau}\right)(\theta+v)
$$

variance, $\sigma^{2}$, is

$$
\left[(\theta+v)^{2}+2 v\right] \Gamma\left(1+\frac{2}{\tau}\right)-\mu_{1}^{\prime}
$$

while the skewness is

$$
\beta_{1}=\frac{\left(\Gamma\left(1+\frac{3}{\tau}\right)\left((\theta+v)^{3}+2 v(3 \theta+3 v+4)\right)-3 \mu_{1}^{\prime} \sigma^{2}+2 \mu_{1}^{\prime 3}\right)^{2}}{\sigma^{6}}
$$

and the kurtosis

$$
\beta_{2}=\frac{\Gamma\left(1+\frac{4}{\tau}\right)\left((\theta+v)^{4}+12 v(\theta+v)^{2}+4 v(8 \theta+11 v+12)\right)-4 \beta_{1}{ }^{1 / 2} \mu_{1}^{\prime} \sigma^{3}-6 \mu_{1}^{\prime 2} \sigma^{2}+6 \mu_{1}^{\prime 3}+5 \mu_{1}^{\prime 4}}{\sigma^{4}} .
$$

Some special cases of chi-square mixture of Weibull distribution:

(1) When $\tau=1$, the chi-square mixture of Weibull distribution reduces to chi-square mixture of exponential distribution with parameters $v$ and $\theta$.

(2) When $\tau=1$, in addition as $v \rightarrow 0$, the limiting distribution of chi-square mixture of Weibull distribution is the exponential distribution with parameter $\theta$.

(3) The limit of a Chi-square mixture of Weibull distribution as $v \rightarrow 0$ is the Weibull distribution with parameters $\tau$ and $\theta$. 
Theorem 2.5 The chi-square mixture of gamma function given by

$$
f(x ; v, \theta, \alpha)=\int_{0}^{\infty} \frac{e^{-\frac{\chi^{2}}{2}}\left(\chi^{2}\right)^{\frac{v}{2}-1}}{2^{\frac{v}{2}} \Gamma\left(\frac{v}{2}\right)} \frac{\left(\frac{x}{\theta+\chi^{2}}\right)^{\alpha} e^{-\left(\frac{x}{\theta+\chi^{2}}\right)}}{x \Gamma(\alpha)} d \chi^{2}, \quad 0<x<\infty
$$

where $v, \theta$ and $\alpha$ are positive real numbers is a probability density function.

Definition 2.3 A random variable $X$ is said to have a chi-square mixture of gamma distribution with $v$ degrees of freedom and parameters $\theta, \alpha$ if it has a probability density function given by (18) above.

Theorem 2.6 Let $X$ be a random variable that follows a Chi-square mixture of gamma distribution with parameters $\theta, \tau$ and $v>0$. Then the rth raw moment about the origin is given by

$$
\mu_{r}^{\prime}=\mathbb{E}\left[X^{r}\right]=\frac{\Gamma(\alpha+r)}{\Gamma(\alpha)} \int_{0}^{\infty} \frac{e^{-\frac{\chi^{2}}{2}}\left(\chi^{2}\right)^{\frac{v}{2}-1}}{2^{\frac{v}{2}} \Gamma\left(\frac{v}{2}\right)}\left(\theta+\chi^{2}\right)^{r} d \chi^{2},
$$

for $r>-\alpha$. Then the mean, $\mu_{1}^{\prime}$, is

variance, $\sigma^{2}$, is

$$
\alpha(\theta+v),
$$

$$
\mu_{1}^{\prime}(\theta+v)+2 v(\alpha+1) \alpha
$$

and the skewness, $\beta_{1}$, is

$$
\frac{\left(\left(\alpha^{3}+3 \alpha^{2}+2 \alpha\right)\left[(\theta+v)^{3}+2 v(3 \theta+3 v+4)\right]-3(\alpha+1)\left[\mu_{1}^{\prime 2}(\theta+v)+2 \mu_{1}^{\prime} \alpha v\right]+2 \mu_{1}^{\prime 3}\right)^{2}}{\sigma^{6}}
$$

Finally the kurtosis, $\beta_{2}$, is equal to

$$
\begin{aligned}
& \left(\frac{\Gamma(\alpha+4)}{\Gamma(\alpha)}\left((\theta+v)^{4}+12 v(\theta+v)^{2}+4 v(8 \theta+11 v+12)\right)\right. \\
& -4 \mu_{1}^{\prime}\left(\frac{\Gamma(\alpha+3)}{\Gamma(\alpha)}\left((\theta+v)^{3}+2 v(3 \theta+3 v+4)\right)\right) \\
& \left.+6 \mu_{1}^{\prime 2}\left(\frac{\Gamma(\alpha+2)}{\Gamma(\alpha)}\left[(\theta+v)^{2}+2 v\right]\right)-3 \mu_{1}^{\prime 4}\right) / \sigma^{4} .
\end{aligned}
$$

Some special cases of chi-square mixture of gamma distribution:

(1) When $\tau=1$, the chi-square mixture of gamma distribution reduces to chi-square mixture of exponential distribution with parameters $v$ and $\theta$.

(2) When $\tau=1$, in addition as $v \rightarrow 0$, the limiting distribution of a Chi-square mixture of gamma distribution is the exponential distribution with parameter $\theta$.

(3) The limit of Chi-square mixture of gamma distribution as $v \rightarrow 0$ is the gamma distribution with parameters $\alpha$ and $\theta$.

Theorem 2.7 The chi-square mixture of transformed gamma function given by

$$
f(x ; v, \theta, \alpha, \tau)=\int_{0}^{\infty} \frac{e^{-\frac{\chi^{2}}{2}}\left(\chi^{2}\right)^{\frac{v}{2}-1}}{2^{\frac{v}{2}} \Gamma\left(\frac{v}{2}\right)} \frac{\tau\left(\frac{x}{\theta+\chi^{2}}\right)^{\tau \alpha} e^{-\left(\frac{x}{\theta+\chi^{2}}\right)^{\tau}}}{x \Gamma(\alpha)} d \chi^{2}, \quad 0<x<\infty
$$

where $\theta, \tau, \alpha$, and $v$ all are positive real numbers is a probability density function.

Definition 2.4 A random variable $X$ is said to have a chi-square mixture of transformed gamma distribution with $v$ degrees of freedom and parameters $\theta, \tau$, and $\alpha$ if its probability density function is given by function (19) above.

The following result is a generalization of the previous results.

Theorem 2.8 Let $X$ be a random variable that follows a chi-square mixture of transformed gamma distribution with $v$ degrees of freedom and positive parameters $\alpha, \tau$ and $\theta$. Then the rth raw moment about the origin is given by

$$
\mu_{r}^{\prime}=\mathbb{E}\left[X^{r}\right]=\frac{\Gamma\left(\alpha+\frac{r}{\tau}\right)}{\Gamma(\alpha)} \int_{0}^{\infty} \frac{e^{-\frac{\chi^{2}}{2}}\left(\chi^{2}\right)^{\frac{v}{2}-1}}{2^{\frac{v}{2}} \Gamma\left(\frac{v}{2}\right)}\left(\theta+\chi^{2}\right)^{r} d \chi^{2}
$$


for $\alpha>-\frac{r}{\tau}$. Thus the mean, $\mu_{1}^{\prime}$, is

$$
\frac{\Gamma\left(\alpha+\frac{1}{\tau}\right)}{\Gamma(\alpha)}(\theta+v)
$$

while the variance, $\sigma^{2}$,

$$
\frac{(\theta+v)^{2}}{\Gamma(\alpha)}\left[\Gamma\left(\alpha+\frac{2}{\tau}\right)-\frac{\left[\Gamma\left(\alpha+\frac{1}{\tau}\right)\right]^{2}}{\Gamma(\alpha)}\right]+\frac{2 v \Gamma\left(\alpha+\frac{2}{\tau}\right)}{\Gamma(\alpha)},
$$

the skewness, $\beta_{1}$, is

$$
\begin{aligned}
& \Gamma(\alpha)\left(\Gamma\left(\alpha+\frac{3}{\tau}\right)\left((\theta+v)^{3}+6 v(\theta+v)+8 v\right)-3 \mu_{1} \Gamma\left(\alpha+\frac{2}{\tau}\right)\left[(\theta+v)^{2}+2 v\right]+2 \mu_{1}^{2}\right. \\
& \left.\Gamma\left(\alpha+\frac{1}{\tau}\right)(\theta+v)\right)^{2} /\left((\theta+v)^{2}\left[\Gamma\left(\alpha+\frac{2}{\tau}\right)-\frac{\left[\Gamma\left(\alpha+\frac{1}{\tau}\right)\right]^{2}}{\Gamma(\alpha)}\right]+2 v \Gamma\left(\alpha+\frac{2}{\tau}\right)\right)^{3}
\end{aligned}
$$

while the kurtosis, $\beta_{2}$, is

$$
\begin{aligned}
& \Gamma(\alpha)\left[\Gamma\left(\alpha+\frac{4}{\tau}\right)\left((\theta+v)^{4}+12 v(\theta+v)^{2}+4 v(8 \theta+11 v+12)\right)-4 \mu_{1}\right. \\
& \left(\Gamma\left(\alpha+\frac{3}{\tau}\right)\left((\theta+v)^{3}+2 v(3 \theta+3 v+4)\right)\right)+6 \mu_{1}^{2}\left(\Gamma\left(\alpha+\frac{2}{\tau}\right)\left[(\theta+v)^{2}+2 v\right]\right) \\
& \left.-3 \mu_{1}^{3} \Gamma\left(\alpha+\frac{1}{\tau}\right)(\theta+v)\right] /\left((\theta+v)^{2}\left[\Gamma\left(\alpha+\frac{2}{\tau}\right)-\frac{\left[\Gamma\left(\alpha+\frac{1}{\tau}\right)\right]^{2}}{\Gamma(\alpha)}\right]+2 v \Gamma\left(\alpha+\frac{2}{\tau}\right)\right)^{2} .
\end{aligned}
$$

Some special cases of chi-square mixture of transformed gamma distribution:

(1) When $\alpha=1$, the chi-square mixture of transformed gamma distribution reduces to chi-square mixture of Weibull distribution with parameters $v, \tau$ and $\theta$. The limit of chi-square mixture of transformed gamma distribution as $v \rightarrow 0$ is the transformed gamma distribution.

(2) When $\tau=1$, the chi-square mixture of transformed gamma distribution reduces to Chi-square mixture of gamma distribution with parameters $v, \alpha$, and $\theta$.

(3) When $\alpha=\tau=1$, the chi-square mixture of transformed gamma distribution reduces to chi-square mixture of exponential distribution with parameters $v$ and $\theta$.

\section{Mixtures of Inverse Transformed Gamma Family}

Proofs are omitted whenever they are similar to proofs given in last section.

Theorem 3.1 The chi-square mixture of inverse exponential distribution function given by

$$
f(x ; v, \theta)=\int_{0}^{\infty} \frac{e^{-\frac{\chi^{2}}{2}}\left(\chi^{2}\right)^{\frac{v}{2}-1}}{2^{\frac{v}{2}} \Gamma\left(\frac{v}{2}\right)}\left(\theta+\chi^{2}\right) \frac{e^{-\frac{\left(\theta+\chi^{2}\right)}{x}}}{x^{2}} d \chi^{2} \quad 0<x<\infty,
$$

where $v$ and $\theta$ are positive real numbers is a probability density function.

Definition 3.1 A random variable $X$ is defined to have a chi-square mixture of inverse exponential distribution with $v$ degrees of freedom and parameter $\theta$ if its probability density function is given by function (20) above.

Theorem 3.2 Let $X$ be a random variable which follows a chi-square mixture of inverse exponential distribution with $v$ degrees of freedom and parameter $\theta$. Then the rth raw moment about the origin is given by,

$$
\mu_{r}^{\prime}=\Gamma(1-r) \int_{0}^{\infty} \frac{e^{-\frac{\chi^{2}}{2}}\left(\chi^{2}\right)^{\frac{v}{2}-1}}{2^{\frac{v}{2}} \Gamma\left(\frac{v}{2}\right)} \cdot\left(\theta+\chi^{2}\right)^{r} d \chi^{2},
$$

which exist for $r>1$.

Hence, a chi-square mixture of inverse exponential has infinite mean (and higher moments), indicating a heavy tail, which is also true for its unmixed distribution, the inverse exponential distribution. 
Note that the the inverse exponential distribution with parameter $\theta$ is the limiting distribution of chi-square mixture of inverse exponential distribution as $v \rightarrow 0$.

Theorem 3.3 The chi-square mixture of inverse Weibull distribution function given by

$$
f(x ; v, \theta, \tau)=\int_{0}^{\infty} \frac{e^{-\frac{\chi^{2}}{2}}\left(\chi^{2}\right)^{\frac{v}{2}-1}}{2^{\frac{v}{2}} \Gamma\left(\frac{v}{2}\right)} \frac{\tau\left(\frac{\theta+\chi^{2}}{x}\right)^{\tau} e^{-\left(\frac{\theta+x^{2}}{x}\right)^{\tau}}}{x} d \chi^{2}, \quad 0<x<\infty
$$

where $v, \theta$, and $\tau$ are positive real numbers is a probability density function.

Definition 3.2 A random variable $X$ is said to have a chi-square mixture of inverse Weibull distribution with $v$ degrees of freedom and parameters $\theta$ and $\tau$ if its probability density function is given by function (21) above.

Theorem 3.4 Let $X$ be a random variable which follows a chi-square mixture of inverse Weibull distribution with $v$ degrees of freedom and parameters $\tau, \theta$. Then the rth raw moment about the origin is given by

$$
\mu_{r}^{\prime}=\mathbb{E}\left[X^{r}\right]=\Gamma\left(1-\frac{r}{\tau}\right) \int_{0}^{\infty} \frac{e^{-\frac{\chi^{2}}{2}}\left(\chi^{2}\right)^{\frac{v}{2}-1}}{2^{\frac{v}{2}} \Gamma\left(\frac{v}{2}\right)}\left(\theta+\chi^{2}\right)^{r} d \chi^{2},
$$

for $\tau>r$. Hence the mean is given by

$$
\mu_{1}^{\prime}=\Gamma\left(1-\frac{1}{\tau}\right)(\theta+v) \quad \text { for } \tau>1,
$$

and the variance

$$
\sigma^{2}=(\theta+v)^{2}\left[\Gamma\left(1-\frac{2}{\tau}\right)-\left(\Gamma\left(1-\frac{1}{\tau}\right)\right)^{2}\right]+2 v \Gamma\left(1-\frac{2}{\tau}\right) \quad \text { for } \tau>2
$$

Furthermore the skewness, $\beta_{1}$, is

$$
\frac{\left(\Gamma\left(1-\frac{3}{\tau}\right)\left((\theta+v)^{3}+2 v(3 \theta+3 v+4)\right)-3 \mu_{1} \Gamma\left(1-\frac{2}{\tau}\right)\left[(\theta+v)^{2}+2 v\right]+2 \mu_{1}^{3}\right)^{2}}{\sigma^{6}} \quad \text { for } \tau>3
$$

while the kurtosis, $\beta_{2}$, is

$$
\begin{aligned}
& \Gamma\left(1-\frac{4}{\tau}\right)\left((\theta+v)^{4}+12 v(\theta+v)^{2}+4 v(8 \theta+11 v+12)\right) \\
& -4 \mu_{1}^{\prime}\left(\Gamma\left(1-\frac{3}{\tau}\right)\left((\theta+v)^{3}+2 v(3 \theta+3 v+4)\right)\right) \\
& +6 \mu_{1}^{\prime 2}\left(\Gamma\left(1-\frac{2}{\tau}\right)\left[(\theta+v)^{2}+2 v\right]\right)-3 \mu_{1}^{\prime 4} / \sigma^{4} \quad \text { for } \tau>4 .
\end{aligned}
$$

Some special cases of chi-square mixture of inverse Weibull distribution:

(1) When $\tau=1$, the chi-square mixture of inverse Weibull distribution reduces to chi-square mixture of inverse exponential distribution with parameters $v$ and $\theta$.

(2) The limit of chi-square mixture of inverse Weibull distribution as $v \rightarrow 0$ is the inverse Wiebull distribution with parameters $\tau$ and $\theta$.

Theorem 3.5 The chi-square mixture of inverse gamma distribution function given by

$$
f(x ; v, \alpha, \theta)=\int_{0}^{\infty} \frac{e^{-\frac{\chi^{2}}{2}}\left(\chi^{2}\right)^{\frac{v}{2}-1}}{2^{\frac{v}{2}} \Gamma\left(\frac{v}{2}\right)} \frac{\left(\frac{\theta+\chi^{2}}{x}\right)^{\alpha} e^{-\left(\frac{\theta+\chi^{2}}{x}\right)}}{x \Gamma(\alpha)} d \chi^{2}, \quad 0<\chi^{2}<\infty
$$

where $\alpha, \theta, v$ are positive real numbers is a probability density function.

Definition 3.3 A random variable $X$ is said to have a chi-square mixture of inverse gamma distribution with $v$ degrees of freedom and parameters $\alpha, \theta$ if its probability density function is given by function (23) above. 
Theorem 3.6 Let $X$ follows a chi-square mixture of inverse gamma distribution with parameters $\alpha, \theta$ and $v$ degrees of freedom. Then the rth raw moment about the origin is given by

$$
\mu_{r}^{\prime}=\frac{\Gamma(\alpha-r)}{\Gamma(\alpha)} \int_{0}^{\infty} \frac{e^{-\frac{\chi^{2}}{2}}\left(\chi^{2}\right)^{\frac{v}{2}-1}}{2^{\frac{v}{2}} \Gamma\left(\frac{v}{2}\right)}\left(\theta+\chi^{2}\right)^{r} d \chi^{2}, \quad \text { for } \alpha>r .
$$

Then the mean and variance are respectively given by

$$
\mu_{1}^{\prime}=\frac{(\theta+v)}{\alpha-1}
$$

and

$$
\sigma^{2}=(\theta+v)^{2}\left(\frac{1+2 v(\alpha-1)}{(\alpha-1)^{2}(\alpha-2)}\right)
$$

Also, the skewness and kurtosis are respectively given by

$$
\beta_{1}=\frac{16\left((\theta+v)^{2}+3 v(\alpha-1)\right)^{2}(\alpha-2)}{(\alpha-3)(\theta+v)^{4}(1+2 v(\alpha-1))^{3}}
$$

and

$$
\begin{aligned}
\beta_{2} & =\left((\theta+v)^{4}+12 v(\theta+v)^{2}+4 v(8 \theta+11 v+12)-4 \mu_{1}^{\prime}(\alpha-4)\left((\theta+v)^{3}+2 v(3 \theta+3 v+4)\right)\right. \\
& \left.+6 \mu_{1}^{\prime 2}(\alpha-3)(\alpha-4)\left((\theta+v)^{2}+2 v\right)-3 \mu_{1}^{\prime 4}(\alpha-1)(\alpha-2)(\alpha-3)(\alpha-4)\right) /\left((\alpha-1)(\alpha-2)(\alpha-3)(\alpha-4) \sigma^{4}\right)
\end{aligned}
$$

Some special cases of chi-square mixture of inverse gamma distribution:

(1) When $\alpha=1$, the chi-square mixture of inverse gamma distribution reduces to chi-square mixture of inverse exponential distribution with parameters $v$ and $\theta$.

(2) The limit of chi-square mixture of inverse gamma distribution, as $v \rightarrow 0$, is the inverse gamma distribution with parameters $\alpha$ and $\theta$.

Theorem 3.7 The chi-square mixture of inversed transformed gamma distribution function given by

$$
f(x ; v, \theta, \alpha, \tau)=\int_{0}^{\infty} \frac{e^{-\frac{\chi^{2}}{2}}\left(\chi^{2}\right)^{\frac{v}{2}-1}}{2^{\frac{v}{2}} \Gamma\left(\frac{v}{2}\right)} \frac{\tau\left(\frac{\theta+\chi^{2}}{x}\right)^{\tau \alpha} e^{-\left(\frac{\theta+\chi^{2}}{x}\right)^{\tau}}}{x \Gamma(\alpha)} d \chi^{2}, \quad 0<x<\infty
$$

where $\theta, \tau, \alpha$, and $v$ all are positive real numbers is a probability density function.

Definition 3.4 A random variable $X$ is said to have a chi-square mixture of inversed transformed gamma distribution with $v$ degrees of freedom and parameters $\theta, \tau$, and $\alpha$ if its probability density function is given by function (24) above.

We have the following result.

Theorem 3.8 Let $X$ follows a chi-square mixture of inverse transformed gamma distribution with $v$ degrees of freedom and parameters $\tau, \theta$ and $\alpha$. Then the rth raw moment about the origin is given by

$$
\mu_{r}^{\prime}=\mathbb{E}\left[X^{r}\right]=\frac{\Gamma\left(\alpha-\frac{r}{\tau}\right)}{\Gamma(\alpha)} \int_{0}^{\infty} \frac{e^{-\frac{\chi^{2}}{2}}\left(\chi^{2}\right)^{\frac{v}{2}-1}}{2^{\frac{v}{2}} \Gamma\left(\frac{v}{2}\right)}\left(\theta+\chi^{2}\right)^{r} d \chi^{2}
$$

for $\alpha \tau>r$. Therefore, the mean and the variance are respectively given by

$$
\mu_{1}^{\prime}=\frac{\Gamma\left(\alpha-\frac{1}{\tau}\right)}{\Gamma(\alpha)}(\theta+v) \quad \text { for } \alpha>\frac{1}{\tau},
$$


and

$$
\sigma^{2}=\frac{(\theta+v)^{2}}{\Gamma(\alpha)}\left[\Gamma\left(\alpha-\frac{2}{\tau}\right)-\frac{\left[\Gamma\left(\alpha-\frac{1}{\tau}\right)\right]^{2}}{\Gamma(\alpha)}\right]+\frac{2 v \Gamma\left(\alpha-\frac{2}{\tau}\right)}{\Gamma(\alpha)} \quad \text { for } \alpha>\frac{2}{\tau},
$$

Similary, the skewness and the kurtosis is also respectively given by

$$
\beta_{1}=\frac{\left(\frac{\Gamma\left(\alpha-\frac{3}{\tau}\right)}{\Gamma(\alpha)}\left((\theta+v)^{3}+2 v(3 \theta+3 v+4)\right)-3 \mu_{1}^{\prime} \frac{\Gamma\left(\alpha-\frac{2}{\tau}\right)}{\Gamma(\alpha)}\left[(\theta+v)^{2}+2 v\right]+2 \mu_{1}^{\prime 3}\right)^{2}}{\sigma^{6}} \quad \text { for } \alpha>\frac{3}{\tau},
$$

and

$$
\begin{aligned}
\beta_{2}= & \frac{\Gamma\left(\alpha-\frac{4}{\tau}\right)}{\Gamma(\alpha)}\left((\theta+v)^{4}+12 v(\theta+v)^{2}+4 v(8 \theta+11 v+12)\right)-4 \mu_{1}^{\prime}\left(\frac{\Gamma\left(\alpha-\frac{3}{\tau}\right)}{\Gamma(\alpha)}\left((\theta+v)^{3}+2 v(3 \theta+3 v+4)\right)\right) \\
& +6 \mu_{1}^{\prime 2}\left(\frac{\Gamma\left(\alpha-\frac{2}{\tau}\right)}{\Gamma(\alpha)}\left[(\theta+v)^{2}+2 v\right]\right)-3 \mu_{1}^{\prime 4} / \sigma^{4} \quad \text { for } \alpha>\frac{4}{\tau} .
\end{aligned}
$$

Some special cases of chi-square mixture of inverse transformed gamma distribution:

(1) When $\alpha=1$, the chi-square mixture of inverse transformed gamma distribution reduces to chi-square mixture of inverse Weibull distribution with parameters $v, \tau$ and $\theta$.

(2) When $\tau=1$, chi-square mixture of inverse transformed gamma distribution reduces to chi-square mixture of inverse gamma distribution with parameters $v, \alpha$ and $\theta$.

(3) When $\alpha=\tau=1$, chi-square mixture of inverse transformed gamma distribution reduces to chi-square mixture of inverse exponential distribution with parameters $v$ and $\theta$.

\section{Results and Conclusions}

In this article, we introduce two models of the chi-square mixture distribution, namely, the chi-square mixture of transform gamma distribution and the chi-square mixture of inverse transform gamma distribution. These models being studied are generalization of the classical transform gamma distribution and inverse transform gamma distribution.

Special submodels of the chi-square mixture of transform gamma distribution, which are deduced and defined, include the chi-square mixture of gamma, Weibull, and exponential mixture distributions. Various properties including mean, variance, skewness, kurtosis are derived. Similarly, out of chi-square mixture of inverse transform gamma distribution, special submodels are also deduced and defined. These are chi-square mixture of inverse gamma, inverse Weibull, and inverse exponential mixture distributions. Also, same statistical properties of each distributions are obtained. Finally, relationship of the two families of chi-square distribution are established via limiting behavior and some special cases.

To best summarized the results, the following figures are helpful for readers understanding. The figure below is for the class of chi-square mixture of transformed gamma distribution.

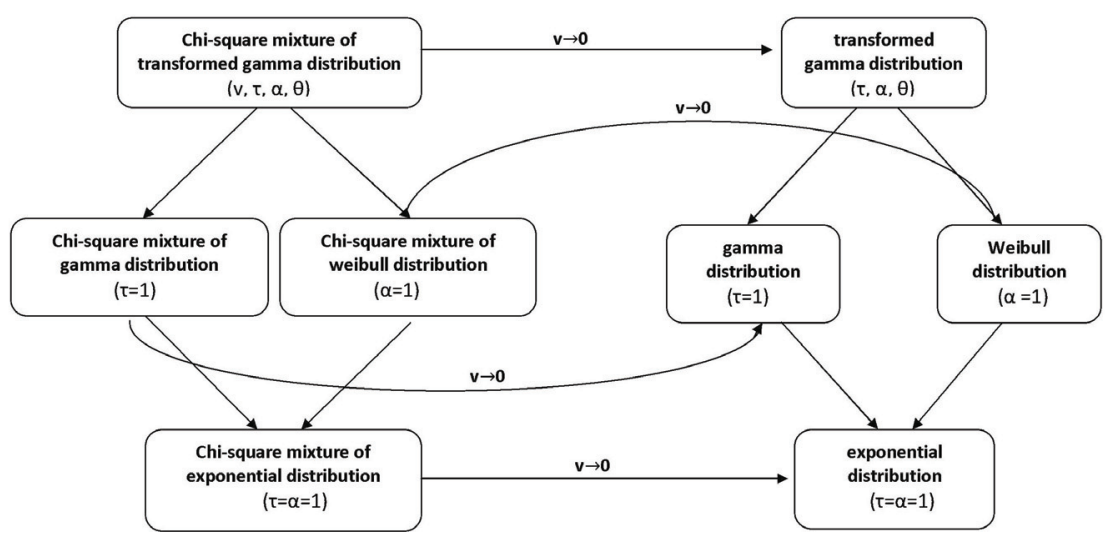

Figure 1. Chi-square mixture of transformed gamma distribution 
Similarly, for the class of chi-square mixture of inverse transformed gamma distribution, we have the figure below.

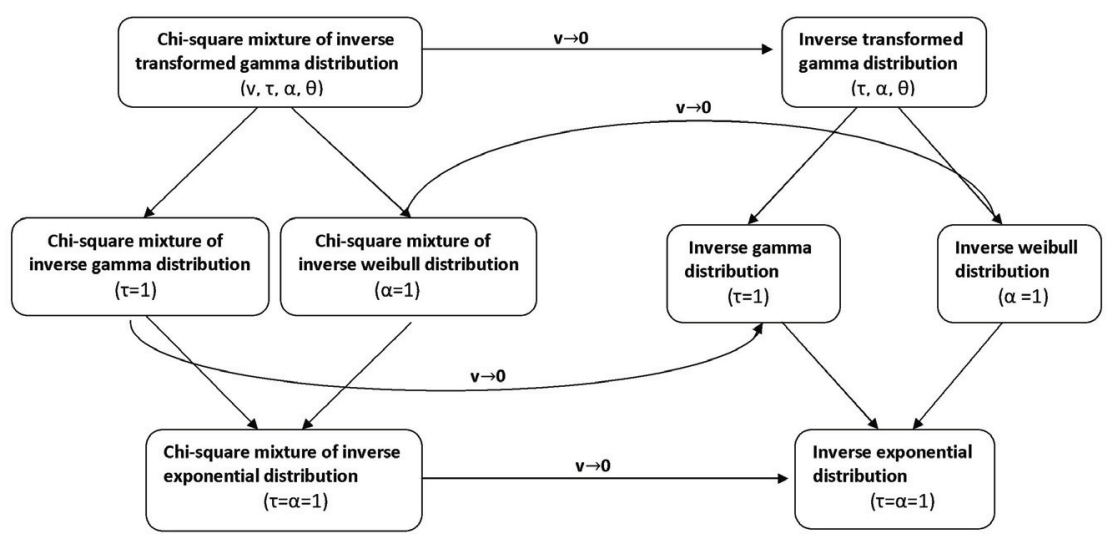

Figure 2. Chi-square mixture of inverse transformed gamma distribution

\section{Acknowledgements}

The authors would like to express their deepest appreciation to the reviewer and editor for their comments and suggestions which lead to the improvement this paper.

\section{References}

Adnan, M. A. S., \& Kiser, H. (2012). A Class of Weibull Mixtured Distributions. Journal of Biometrics and Biostatistics, 3, 135. http://dx.doi.org/10.4172/2155-6180.1000135

Alexander, C. (2004). Normal mixture diffusion with uncertain volatility: Modelling short- and long-term smile effects. Journal of Banking \& Finance, 28(12), 2957-2980. http://dx.doi.org/10.1016/j.jbankfin.2003.10.017

Allison, D. B., Gadbury, G. L., Heo, M. S., Fernandez, J. R., Lee, C. K., Prolla, T. A., \& Weindruch, R. (2002). A mixture model approach for the analysis of microarray gene expression data. Computational Statistics \& Data Analysis, 39, 1-20. http://dx.doi.org/10.1016/S0167-9473(01)00046-9

Baral, N., Nagy, C., Crain, B. J., \& Gautam, R. (2013). Population viability analysis of Critically Endangered white-rumped vultures Gyps bengalensis. Endangered Species Research, 21, 65-76. http://dx.doi.org/10.3354/esr00511

Böhning, D., Seidel, W., Alfó, M., Garel, B., Patilea, V., \& Walther, G. (2007). Advances in Mixture Models. Computational Statistics and Data Analysis, 51, 5205-5210.

Coli, N., Boldini, D., \& Bandini, A. (2012). Modeling of complex geological rock mixtures under triaxial testing conditions. Eurock, Stockholm.

Dempster, A. P., Laird, N. M., \& Rubin, D. B. (1977). Maximum likelihood estimation from incomplete data via the EM algorithm (with discussion).

Dong, Z. (1997). Mixture Analysis and its Preliminary Application in Archaeology. Journal of Archaeological Science, 24, 141-161. http://dx.doi.org/10.1006/jasc.1996.0100

Fleischman, S. J., \& Burwen, D. L. (2003). Mixture models for the species apportionment of hydroacoustic data, with echo-envelope length as the discriminatory variable. ICES Journal of Marine Science, 60, 592-598. http://dx.doi.org/10.1016/S1054-3139(03)00041-9

Gutierrez, R. G., Carroll, R. J., Wang, N., Lee, G.-H., \& Taylor, B. H. (1995). Analysis of tomato root initiation using a normal mixture distribution. Biometrics, 51, 1461-1468.

Hunt, G., \& Chapman, R. E. (2001). Evaluating hypotheses of instar-grouping in arthropods: a maximum likelihood approach. Paleobiology, 27(3), 466-484.

Lindsay, B. G. (1995). Mixture models: theory, geometry and applications. NSF-CBMS Regional Conference Series in Probability and Statistics, 5. Institute of Mathematical Statistics, Hayward California. American Statistical Association, Alexandria, Virginia. 
Liu, S., Li, S., Zhang, L., \& Wu, Y. (2008). A mixture model with modified mass transfer expression for cavitating turbulent flow simulation. Engineering Computations, 250-304. http://dx.doi.org/10.1108/02644400810874930

Macdonald, P. D. M. (1987). Analysis of length-frequency distributions. In R. C. Summerfelt \& G. E. Hall (eds.), Age and Growth of Fish (pp. 371-384). Ames, Iowa: Iowa State University Press.

Melnykov, V., Maitra, R., \& Nettleton, D. (2011). Accounting for Spot Matching Uncertainty in the Analysis of Proteomics Data from Two-Dimensional Gel. Electrophoresis, Sankhya: Series B, 73(1), 123-143. http://dx.doi.org/10.1007/s13571-011-0016-x

Panjer, H. (2006). Operational Risk: Modeling Analytics. New York: Wiley. http://dx.doi.org/10.1002/0470051310

Pearson, K. (1894). Contribution to the mathematical theory of evolution. Philosophical Transactions of the Royal Society A., 185, 71-110.

Ram, N., \& Grimm, K. J. (2009). Growth mixture modeling: A method for identifying difference in longitudinal change among unobserved groups. International Journal of Behavioral Development, 33, 565-576.

Rezaul, K., Pear, H., Sultana, B., \& Forhad, H. (2011). Rayleigh Mixture Distribution. Journal of Applied Mathematics. http://dx.doi.org/10.1155/2011/238290

Roy, M. K., Zaman, M. R., \& Akher, N. (2005). Chi-square mixture of Gamma Distribution. Journal of Applied Sciences, 5(9), 1632-1635.

Roy, M. K., Zaman, M. R., \& Akher, N. (2006a). Chi-square mixture of Chi-square Distributions. Journal of Applied Sciences, 6(2), 243-246.

Roy, M. K., Zaman, M. R., \& Akher, N. (2006b). Chi-square mixture of Erlang Distribution. Trends in Applied Science Research, 1(5), 487-495.

Schlattmann, P. (2009). Medical Applications of Finite Mixture Models (Statistics for Biology and Health). Berlin: Springer Verlag. http://dx.doi.org/10.1007/978-3-540-68651-41

Schork, N. J., Allison, D. B., \& Theil, B. (1996). Mixture distributions in human genetics research. Statistical Methods in Medical Research, 5(2), 155-178. http://dx.doi.org/10.1177/096228029600500204

Sylvester, Z. (2007). Turbidite bed thickness distributions: methods and pitfalls of analysis and modelling. Sedimentology, 54(4), 847-870. http://dx.doi.org/10.1111/j.1365-3091.2007.00863.x

Xu, L., Hanson, T., Bedrick, E., \& Restrepo, C. (2010). Hypothesis tests on mixture model components with applications in ecology and agriculture. Journal of Agricultural, Biological, and Environmental Statistics, 15(3), 308-326. http://dx.doi.org/10.1007/s13253-010-0020-z

Yang, Z. R., \& Zwolinski, M. (2011). Mutual information theory for adaptive mixture models. IEEE Transactions on Pattern Analysis and Machine Intelligence, 23(4), 396-403. http://dx.doi.org/10.1109/34.917574

\section{Copyrights}

Copyright for this article is retained by the author(s), with first publication rights granted to the journal.

This is an open-access article distributed under the terms and conditions of the Creative Commons Attribution license (http://creativecommons.org/licenses/by/3.0/). 